\title{
Using the Point Cloud Data to Reconstruct CAD Model by 3D Geometric Modeling Method in Reverse Engineering
}

\author{
Yan-qin Li ${ }^{1}$, Gui-zhong Xie ${ }^{1}$,Fan-nian Meng ${ }^{1}$, De-hai Zhang ${ }^{1,2}$, * \\ ${ }^{1}$ Mechanical and Electrical Engineering Institute, Zhengzhou University of Light Industry, China \\ ${ }^{2}$ State Key Laboratory for Manufacturing Systems Engineering (Xi'an Jiaotong University), China \\ *zhangdehai0318@163.com
}

Keywords: Geomagic, Point cloud, CAD, Reverse Engineering.

\begin{abstract}
Reverse engineering (RE) is widely used on the aspects about designing of advanced products, software or images. The 3D Geometric modeling method is composed of measurement equipment and RE software. The three-dimensional digital measurement equipment is used to collect the model's point cloud data and reconstruct CAD model. Geomagic Studio is widely used to process data and repair models, while Geomagic Qualify is widely used in testing of reconstruction model. The point cloud data of a turbine blade is collected and processed, some technologies and steps including pre-processing of point cloud data, polygon processing, surface fitting, triangular mesh, model smoothing and polygon mesh optimization are conducted. Finally, it is being demonstrated that 3D Geometric modeling method in Reverse Engineering is successful.
\end{abstract}

\section{Introduction}

According to the reverse object we used, RE can be divided into three kinds including of physical reverse, software reverse and image reverse. At present, much of the reverse engineering technology research are concentrated in geometry shape, reconstruct CAD model to repair products, it is different with the forward process that from drawings to products and RE is the process that material-drawing-parts [1]. The three-dimensional digital measurement equipment is used to collect the model's point cloud data and these point cloud data is used to reconstructing CAD model by 3D geometric modeling method.

The core of the RE technology is measuring equipment and processing software. Measuring equipment PIX-30 Dot Scanner, CYCLON2 High Speed Scanner, TALYSCAN150 Multi-sensor Scanner and ATOS scanner are widely used. The representatives of RE software are Geomagic Studio, Image wave, Rapidform, CATIA, etc. Those Pro/SCAN in the Pro/E and Point Cloud function module in the UG, are used to process parts and product model. These tools can be selected according to the actual needs and the complexity and accuracy of the physical objects. Geomagic Studio is widely used to process data and repair models. Geomagic Qualify is widely used in testing of reconstruction model.

\section{The Preprocessing Of Point Cloud Data, Polygon Processing And Surface Fitting}

Whether it is a non contact type or contact type, point cloud data of measurement model are equipped with large number of discrete points. These factors including of external light, environmental impact and condition of measurement constraints will affect quality of point cloud data. For example, some abnormal points, the discrete data can make the object shape feature fuzzy and the expression is unclear and so on. In order to resolve these problems, it requests us to conduct the data memory processing. In the process of scanning, due to the light interference, the staff of shaking, the surface of some impurities which will bring the mixed point and noise points. The pre-processing of data and polygon processing is the necessary step in improving data quality [2]. 


\section{Point Cloud Removal}

During the data collection, some big error points will happen due to the influence of environment and measurement system error. The noise points can be caused by the vibration of the components in the rotation, there is a rough surface, or when the scan, beyond the boundaries of the work piece, the other things into the scan. These points are not needed, we can see the free point cloud by the computer amplification, for the existence of large do not need to point cloud, we can manually delete and other points can be used in Geomagic Studio Software, non connection option will be deleted those red and bright spots when you can click the delete Tab. The untreated point cloud of turbine blade is shown in the Figure 1 (a), the treated noise processing as shown in the Figure 1 (b). By contrast the following picture, the noise has been removed. Each model surface quality has been improved largely [3].
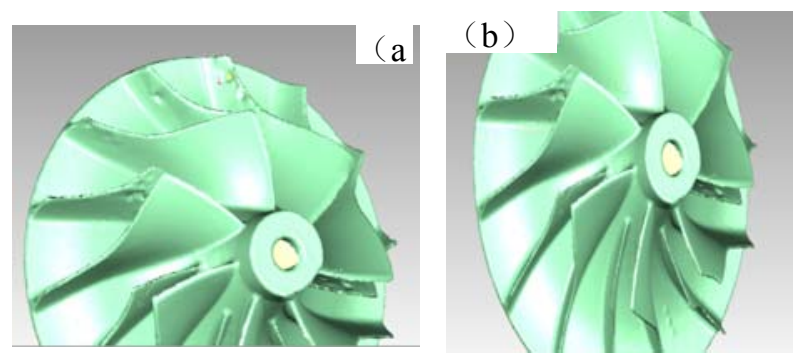

Fig. 1 Comparison of point clouds before and after removal, a) treated colored point cloud, b) the colored point cloud after removing the speck
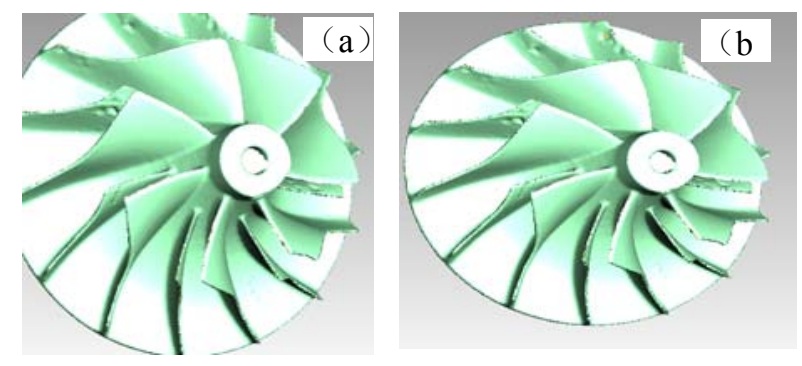

Fig. 2 Contrast before and after sampling, a) point cloud data before sampling, b) sampled point cloud data

\section{Other Methods for Pre-processing of Point Cloud Data}

In addition to the above several methods of point cloud data pre-processing, but also includes other important way, sometimes, in order to improve the quality of point cloud data pre-processing, we should learn to utilize different treatment methods for the different point cloud situations.

Data Reduction. A huge amount of point cloud data will bring trouble to the later model reconstruction, for example, treatment of slow, low efficiency, high requirements for hardware, in order to ensure the quality of the reconstructed model[4], we should reduce the number of measurement points as far as possible, to lay the foundation for future treatment. For example, the random sampling method, the uniform grid method [5] and the triangle grid method [6] can be selected for the scattered points.

Data Smoothing. After the noise points are removed, the use of data smoothing reduces the subsequent calculation error, so it's very good to improve the modeling accuracy.

Feature Extraction. The extraction of geometric features of the object surface provides a key support for the subsequent model reconstruction, which ensures the reconstruction of the geometric shape, and ensures the geometric features of the reconstruction in a reasonable error range, and ensures the accuracy of the model.

Coordinate Transformation. In the next operation processing, a reasonable measurement point of view can improve the efficiency and accuracy of the operation.

Because some of the examples of this subject are obtained by using the hand-held 3D laser scanning, Streamlining of point cloud data in Geomagic Studio is to use the "unified sample" command, absolute pitch is set to $0.1 \mathrm{~mm}$, the system will follow the set point margin of sampling, sampling point cloud data a lot, and the model did not affect its characteristics. Sample this process will further filter a few overlapping points. As shown in Figure 2 before and after sampling. 


\section{The Triangular Mesh of Point Cloud Data}

Before all operations are in point, point display parts of the visualization is poor, it is difficult to distinguish between the external characteristics of the point cloud model, we will point cloud data triangulation is in order to more clearly the external characteristics of a more intuitive observation model, point cloud polygon can get better effect.

The data points are connected into a triangle is a triangular grid point cloud data. In this way, we can turn a point cloud model into a polygonal mesh model. At present, the Delaunay triangulation method is the most common, according to the difference of the realization method, the method of Delaunay triangulation can be divided into three kinds.

In Geomagic Studio software, the "encapsulation" command to point cloud data triangulation, maximum number of triangles set to 350000 , this is encapsulated triangle maximum number, executive encapsulation command, the system will automatically calculate the number of triangles, using triangular mesh model data display, Figure 3 (a) is package model, Figure 3 (b) is partial enlargement, can see the point cloud is a triangular mesh.

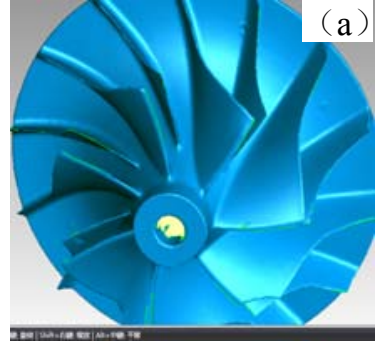

Fig. 3 Model point cloud triangulation a) point cloud triangular mesh model, b) local triangular mesh
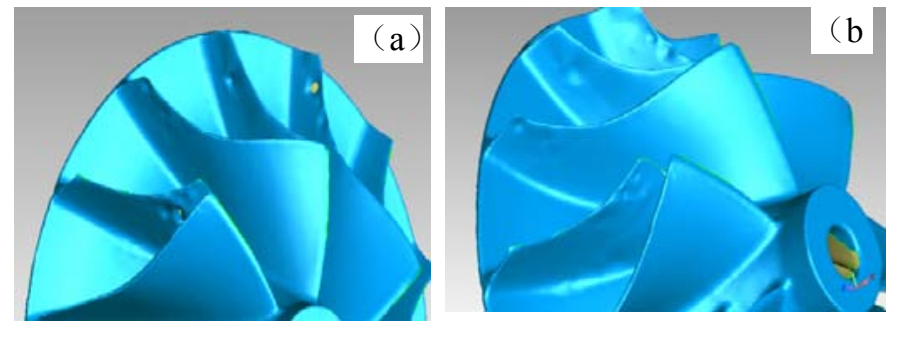

Fig.4 Filling effect contrast diagram

\section{Polygon Mesh Optimization}

Through the triangle mesh models of, poor surface finish can be seen, there is missing data, for example, no scanning, leaving holes, there are convex concave surfaces and so on, where you need to use the software to model data filling and smoothing treatment, or after in order to facilitate the operation.

Data Repair. These models using 3D laser scanning, may exist without scanning to place, resulting in lack of a small part of the data, treatment need to fill a vacancy on the point cloud data. In Geomagic Studio software with "fills holes" to fill missing data command separately for each model, encounter point cloud data duplication and irregular triangles, then you want to delete this type of triangle, delete and then fill these missing data to smooth the model. Filling the hole, should mainly consider the change of curvature around the feature model by filling hole method based on curvature, as shown in Figure 5, fill Effect contrast diagram before and after filling:

\section{Polygon Model Smoothing}

After you fix the hole on the surface of some parts of the poor finish, smoothing treatment is needed. In order to make high efficiency after, to model the overall features and details are not affected under the premise; reducing the number of triangles, need to use the software in the "simplified polygon" command to reduce the number of triangles. In simplified polygonal section bar set "reduced to a percentage" is $85 \%$, on behalf of the number of triangles has reduced by $15 \%$, Next step is to create a stream, the bulging and irregular triangle sanding, grinding intensity is moderate, too much will cause local deformation in the model, then can also use: remove features, sandpaper, remove the spikes with the use of command etc. Removing object itself does not exist the bump and irregular triangle, so that the parts of the surface more smooth, triangular mesh is smoother, finally in 
"polygon relaxation" command, adjusting the included angle of the triangle anti wrinkle is object is more fairing. After the relaxation of the model, the deviation of the model shows that the chromatogram is shown in the Figure 5.

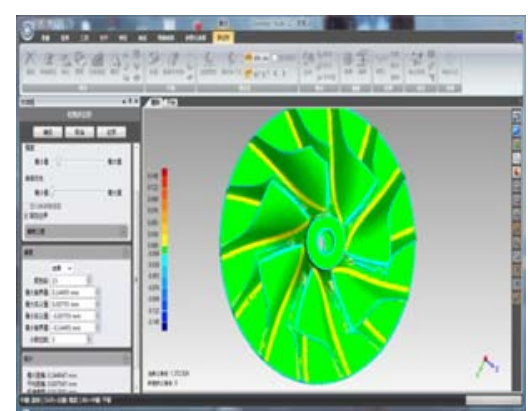

Deviation display chromatogram
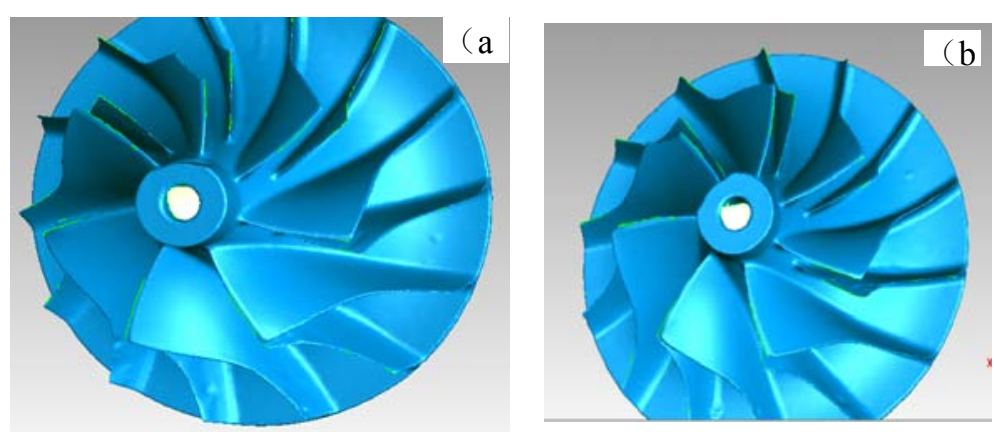

Fig.6 Contrast diagram of smooth processing before and after processing a) smoothing before b) after smoothing

By the above deviation display chromatogram can be seen, the maximum deviation was 0.145 , the minimum deviation is 0.145 , this shows that after smoothing the model error is relatively small, relaxed further after repair of irregular triangle until the entire polygon is relatively smooth. Figure 6 is before and after smoothing the comparison chart:

\section{Conclusions}

The 3D Geometric modeling method is composed of measurement equipment and RE software. The three-dimensional digital measurement equipment is used to collect the model's point cloud data and reconstruct CAD model. Geomagic Studio is widely used to process data and repair models, while Geomagic Qualify is widely used in testing of reconstruction model. The point cloud data of a turbine blade is collected and processed, some technologies and steps including pre-processing of point cloud data, polygon processing, surface fitting, triangular mesh, model smoothing and polygon mesh optimization are conducted. Finally, it is being demonstrated that 3D Geometric modeling method in Reverse Engineering is successful.

\section{Acknowledgement}

This research was financially supported by the State Key Laboratory for Manufacturing Systems Engineering ( $\mathrm{Xi}$ ' an Jiaotong University) [Grant Number sklms2015012], the Key Scientific Research Project of Henan Province [Grant Number 142102110151], Production-study-research Cooperation Project of Henan Province [Grant Number 162107000045], the Doctoral Program of Zhengzhou University of Light Industry.

\section{References}

[1] D. H. Zhang, J. Liang, C. Guo, J. W. Liu, X. Q. Zhang, Z. X. Chen, Exploitation of photogrammetry measurement system, Opt. Eng. 49 (2010)037005.

[2] S. J. Zhang, Y. R. Liu, Y. Nie, Research review of dissolving natural polymer materials with ionic liquids and green spinning technology, J. Light Indus. 31 (2016)1-14.

[3] D. H. Zhang,Y. Q. Li, J. X. Liu, G. Z. Xie, E. W. Su, A novel 3D optical method for measuring and evaluating springback in sheet metal forming process, Measurement, 92 (2016) 303-317.

[4] D. H. Zhang, G. Z. Xie, Y. Q. Li, J. X. Liu, Strain and mechanical properties of VCM multi-layer sheet and their composites using digital speckle correlation method, Appl. Optics. 54 (2015) 7534-7541. 
[5]D. H. Zhang, D. P. Bai, J. B. Liu, Z. Guo, C. Guo, Formability Behaviors of 2A12 Thin-wall Part Based on DYNAFORM and Stamping Experiment, Compos. Part B. 55(2013) 591-598.

[6] D. H. Zhang, J. Liang, C. Guo, Z. X. Chen, Integrated precision evaluation method for 3D optical measurement system, P. I. M. Eng. Part B-J. Eng. Manuf. 225 (2011), 909-920. 